\title{
Posterior limbus vertebral lesions causing lumbosacral radiculopathy and the cauda equina syndrome
}

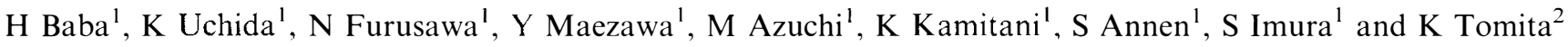 \\ ${ }^{1}$ Department of Orthopaedic Surgery, Fukui Medical School, Shimoaizuki 23, Matsuoka, Fukui 910-11, Japan; \\ ${ }^{2}$ Department of Orthopaedic Surgery', School of Medicine, Kanazawa University, Takaramachi 13-1, Kanazawa 920, \\ Japan
}

\begin{abstract}
This report reviews our experience with spinal decompression for posterior limbus vertebral lesions or osteocartilaginous vertebral corner defects in the lumbar spine in 29 children and young adults. There were 19 male and 10 female patients with a mean age of 16.5 years (range, 9 to 24 years). Twenty-four patients were involved with various athletic activities. Clinical presentation included low back pain with a variable degree of radiculopathy in 25 patients and a cauda equina syndrome in four. The level of the affected spinal area was L1-2 in one patient, L2-3 in one, L3-4 in seven, L4-5 in 17, and L5-S1 in three. The preoperative imaging workup showed lateralised 'non-calcified' or 'calcified' limbus vertebral defects in 13 patients and centrally displaced lesions in 16 patients. All patients underwent posterior spinal decompression with a slightly extended laminotomy, except for three patients who had a subsequent posterolateral fusion. All of the patients consequently returned to practice their favourite preoperative sport and lifestyle, but five discontinued their previous sports. We suggest that patients with posterior limbus vertebral lesions require careful diagnosis and therapy that are different from those with an ordinary lumbar disc herniation.
\end{abstract}

Keywords: radiculopathy; cauda equina syndrome; apophyseal ring; osteocartilaginous endplate; gymnastic trauma; lumbar spine

\section{Introduction}

Limbus vertebral bulge in the lumbosacral spine can cause symptoms and signs of radiculopathy and the cauda equina syndrome in young patients. This osteocartilaginous pathology may develop as an acute process following trauma of the posterior vertebral corner in vigorous physical exercise. The clinical presentation is essentially similar to that complicating a herniated intervertebral disc, but may be more significant because of the displaced mass lesion occupying a wide region anteriorly within the spinal canal. Treatment, including surgical decompression, may thus be different from that employed routinely for those with a lumbar disc herniation.

The present communication describes our experience of patients with symptomatic limbus vertebral lesions and posterior apophyseal ring fractures requiring surgical decompression.

\section{Patients and methods}

Patients and evaluation

The present study included a total of 29 surgical series presenting with neurological symptoms and signs

Correspondence: H Baba pertaining to the lumbosacral spine associated with limbus vertebral lesions and with posterior apophyseal ring fractures. The mean age at surgery was 16.5 years (range 9 to 24 years), and included 19 males and 10 females. The duration of symptoms varied widely from 3 days to 5.5 months (average, 1.3 months) before admission to our two Medical Centres. A number of patients were involved in a variety of gymnastic exercises and the severity of the neurological complications varied from one patient to another. Following a written informed consent, all patients subsequently underwent a posterior spinal decompression. Follow-up periods ranged from 1.3 to 9.5 years, with an average of 5.8 years.

The postoperative clinical presentations and physical activities were rated into four grades: grade A (excellent), no abnormal neurological findings and the subject reported an active preoperative lifestyle including sport; grade B (good), minimal persistence of radicular symptoms and neurological complications that did not restrict preoperative social activities, but limited previous athletic engagements; grade C (fair), persistent pain and neurological compromise that obviously affected previous physical as well as social activities; and grade D (poor), severe neurological symptoms that required readaptation to social activity and discontinuation of previous gymnastic activity or worsening of performance. 
In addition to plain radiographs, the imaging workup included myelography in 13 patients, computed tomography (CT) in 28 , and magnetic resonance imaging (MRI) scan in 20. Electromyography was indicated in eight cases who presented with weakness of the muscles of the lower extremities, and cystometry in five patients. The limbus vertebral lesions were classified into five subtypes according to the radiological classification described by Epstein:' type I, central avulsion of the posterior cortical rim; type II, central avulsion of posterior cortical rim with a cancellous fragment; non calcified (NC) type III, lateralised 'non calcified' chip lesion of the posterior cortical rim mimicking disc herniation; calcified (C) type III, lateralised 'calcified' chip lesion of the posterior cortical rim; and type IV. central avulsion of the posterior cortical rim with a cancellous bone fragment extending across the entire vertebral body between the endplates.

Radiologically, we followed patients carefully to monitor the development of any clinical hypermobility (instability), and/or spondylolisthesis especially anteriorly, at the operated segments. In 26 patients without fusion (12 with unilateral and 14 bilateral laminotomy), flexion-extension lateral radiographs were viewed up to 9.5 years (average 6.1 years) after surgery. Using a modified check list of White and Panjabi $^{2}$ describing clinical instability, the operated segment was clinically unstable when the sagittal plane translation of the vertebra exceeded $4.0 \mathrm{~mm}$ (anterior spondylolisthesis) and/or the anterior sagittal plane translation increased by more than 15 degree (hypermobility) on follow-up radiographs. In three patients with one-level posterolateral fusion, the solid fusion mass was evaluated by anteroposterior radiographs. In eight cases with hamstrings tightness $(n=6)$ or motor deficits $(n=2)$, further MRI studies were necessary to view residual compressive lesions and/or wound haematoma.

\section{Surgical technique}

The operation included unilateral or bilateral partial laminotomy (fenestration) with or without posterolateral fusion at the affected spinal level. Using a standard posterior midline approach, the affected nerve root and the dura mater were exposed through an extended laminotomy window of the distal part of the lamina proximally until the attachment of the ligamentum flavum was identified and resected. The proximal part of the caudal lamina was also partially resected to view safely the so-called 'axilla' of the affected nerve root. When the lesion extended laterally within the spinal canal and impinged on the nerve root at the entrance of the intervertebral foramen, a small medial foraminotomy was supplemented with a microsurgical technique. No more than one half of the facet joint was basically injured during the interlaminar fenestration and foraminotomy in order to avoid subsequent need for fusion, except in three patients. Following careful dissection of the neural tissue from the mass located anteriorly and unilaterally or bilaterally, the limbus vertebral lesion was excised en bloc or in piecemeal. The compressive lesion was resected so that the affected nerve root and the dura mater were completely decompressed. In cases with coexisting annulus fibrosus extrusions subligamentously or transligamentously, exhaustive search for fragmented disc materials was also performed. Posterolateral fusion with autogenous bone grafting was performed in three patients who needed more than $50 \%$ medial facetectomy (two patients) or had a significant hypermobility (instability) preoperatively at the level of involvement (one patient). The routine regimen of postoperative care consisted approximately 10 days bed rest followed by ambulatory rehabilitation with a spinal brace for 3 weeks. The return to practising a favourite sport over a period of time depended on the level of each athletic activity.

\section{Results}

Spinal level involved and type of compressive lesion The L4-5 level was the most frequently involved site followed by L3-4. The spinal level affected included L12 in one patient, L2-3 in one, L3-4 in seven, L4-5 in 17 and L5-S1 in three patients. One child showed a large displaced osteocartilaginous mass between L5-S1 level and S2 segment, occupying a large section of the lumbosacral spinal canal (Figure $1 \mathrm{a}, \mathrm{b}$ and $\mathrm{c}$ ). The radiological appearance of limbus vertebral lesion included type I in eight patients, type II in seven, type NCIII in five, type CIII in eight and type IV in one patient.

\section{Clinical presentation and radiologic follow-up}

Clinical presentation according to type of limbus vertebral lesion is shown in Table 1. Hamstrings tightness and mechanical signs, such as positive Laségue test and femoral nerve stretch test, were frequently observed. Sensory disturbances were noted in $11(41 \%)$ patients, motor deficits in eight $(28 \%)$, and neurogenic claudication with variable walking distance in $21(72 \%)$.

Postoperatively, all patients recovered smoothly from radicular symptoms and the cauda equina complications. The period between surgery and improvement of sensory and motor deficits to acceptable levels, ranged from 2 to 15 weeks (average, 5.5 weeks), and to regain normal sense of urination, 5.5 weeks. The resolution of hamstrings tightness occurred after a relatively long period after surgery (average, 6.4 months; range 3.5 to 9.8 months).

The functional outcome was assessed as grade $\mathrm{A}$ in 21 patients and grade $\mathrm{B}$ in eight cases. Twenty-four patients were engaged in various types of athletic activities preoperatively, 19 of these patients practised their sport activity postoperatively while five volunta- 


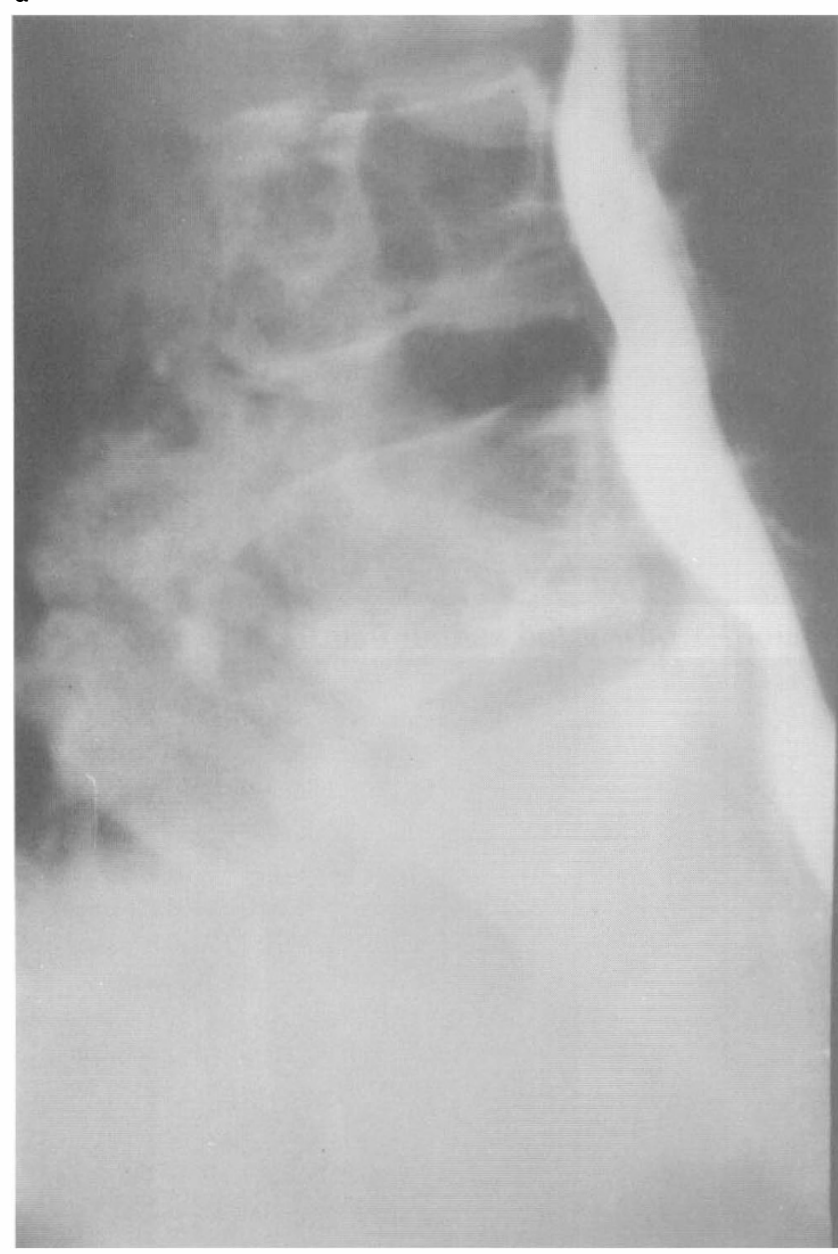

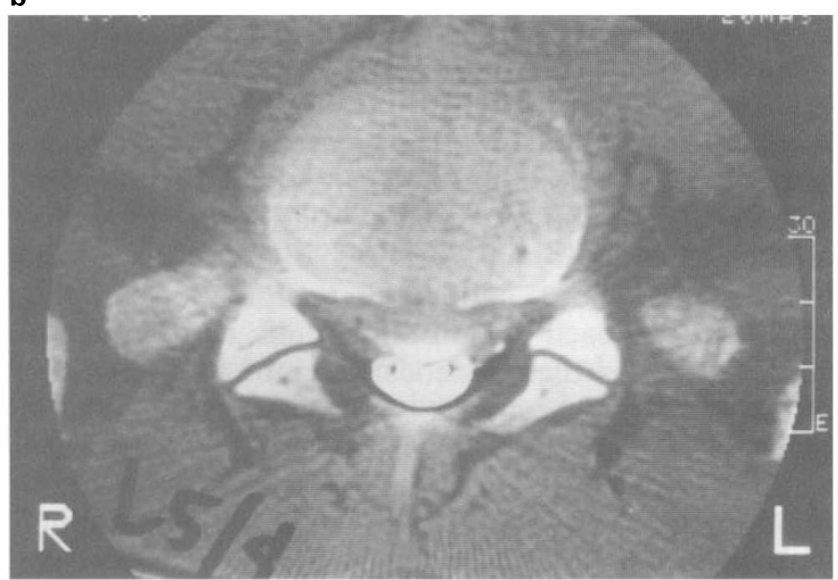

c

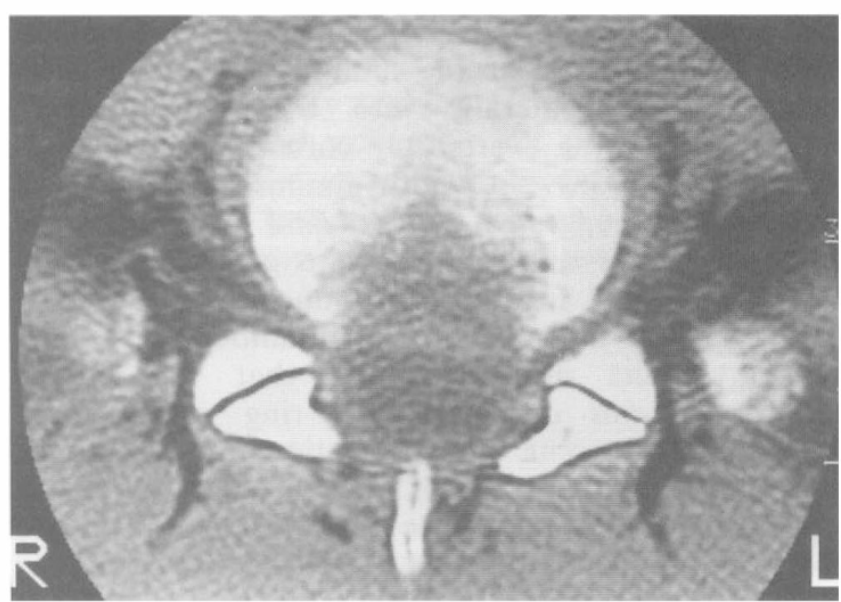

Figure 1 A 10-year-old boy with a 2-year history of trampoline exercise. (a) preoperative myelogram showing avulsed posterior vertebral cortex of S1 (type IV lesion); (b) preoperative computed tomography (CT) scan at L5-S1 level; and (c) postoperative CT scan at the same level

rily changed their sport to practice a different less physically demanding sport activity. Three of these 24 patients were national and semi-national class junior gymnasts (one in Kayak, one in Judo and one in Bicycle), of whom two consequently returned to their sports. A 15-year-old boy, who was a nominated trainee of the National Judo Association, required approximately 2.7 months to recover from the acute onset of symptoms of the cauda equina syndrome, but required 1.2 years to regain his previous ability to be an outstanding junior Judo wrestler (Figures $2 \mathrm{a}, \mathrm{b}$ and c).

All three patients with single-level posterolateral fusion, including one for L3-4 level and two for L4-5, achieved solid bony union 8 months after surgery. These patients did not develop any instability (hypermobility) or spondylolisthesis above or below the site of fusion in the lastest follow-up. Furthermore, none of 26 patients with a unilateral or bilateral extended laminotomy demonstrated clinical hypermobility or spondylolisthesis in the operated segments at final follow-up. In eight patients who required MRI postoperatively, including six patients with hamstrings tightness (one with type II, three with type NCIII and two with type CIII) and two patients with motor deficits (type IV), none showed evidence of significant osteocartilaginous compression or wound haematoma on MRI.

\section{Surgical complications}

Surgery-related minor complications occurred in three cases. Two patients reported temporary painful paraesthesia in the dermatome innervated by the affected nerve root, including one in L3 dermatome and another in L4. In the series there was no recurrence at the operated site or development of a similar disorder in the other spinal levels. 


\section{Discussion}

Lumbar disc herniation in children and adolescents as well as in young adults is not rare. However, neurological complications associated with a displaced posterior vertebral limbus or a fractured apophyseal ring are uncommon. ${ }^{1,3-6}$ Differentiation between these pathological conditions is possible and, due to the different modalities of neural compression, specific treatment for each condition should be considered. Furthermore, as these osteocartilaginous lesions occur particularly in association with excessive gymnastic exercises, surgical treatment should be performed cautiously so as to allow complete recovery without residual neurological dysfunction and the continuation of the gymnastic and social activities.

Displacement of the osteocartilaginous endplate is an avulsion fracture caused by the dislodgement of the posterior longitudinal ligament. The ligament covers a wide area of the annulus fibrosus posteriorly, and far laterally into the neuroforamen. However, at the vertebral corner where it is attached, the transverse width diminishes rapidly. As was correctly suggested by Ehni and Schneider, ${ }^{7}$ the vertebral corner and the endplate are mechanically weak, especially for a distraction force, especially during flexion movement of the lumbosacral spine. These workers also explained that the limbus vertebral lesions or apophyseal ring avulsion are more likely to occur than disruption of the annulus fibrosus (posterior Sharpey's fibres) and the posterior longitudinal ligament, when the flexion forces are abruptly applied in the middle to the lower lumbar spine regions. Savini et $a l^{5}$ have described that posterior lumbar apophyseal lesions occur in children as a result of a single or of repeated trauma at the junction of the cartilage endplate and the ossified portion of the vertebral body. Although it is uncertain how the mechanical stress occurred in each individual in the present study, 24 of 29 patients were involved in physically demanding sport activity; and only 10 of 24 cases reported certain events that caused the sudden appearance of symptoms. We could not find any relationship between the degree and type of physical activity and type and grade of osteocartilaginous lesion. However, we believe that excessive mechanical stress was applied to the lumbar spine and contributed to the development of the lesion.

Symptoms and signs associated with a limbus vertebral lesion is primarily similar to those of a herniated intervertebral disc. However, our experience, similar to that of Takata et $a l^{8}$ suggest that hamstrings tightness and mechanical tension signs are prominent. Interestingly, the hamstrings tightness lasted for a considerably longer time postoperatively despite satisfactory surgical decompression. In general, the nerve root and the cauda equina of a child show increased tension longitudinally partly in accordance with the 'ascensus medullaris'. This characteristic may

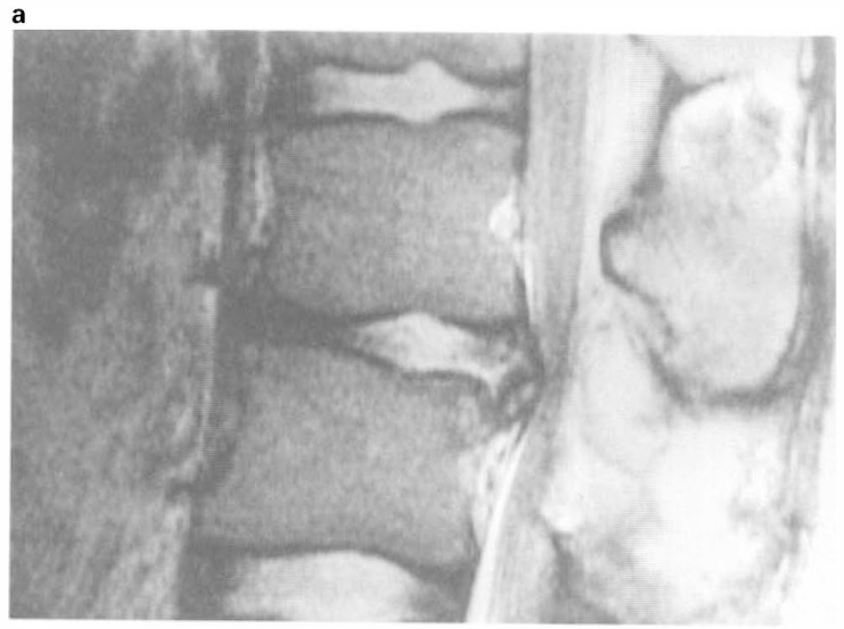

b

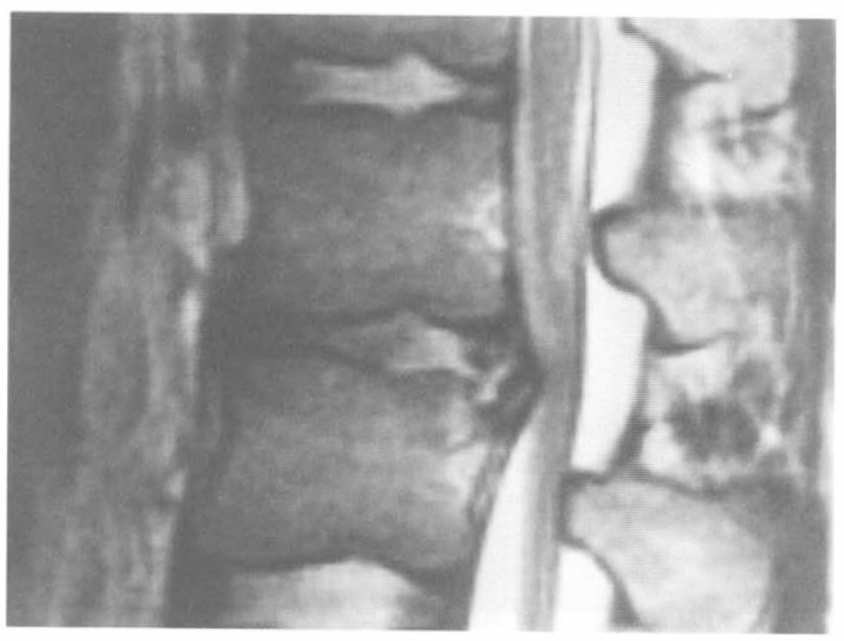

c

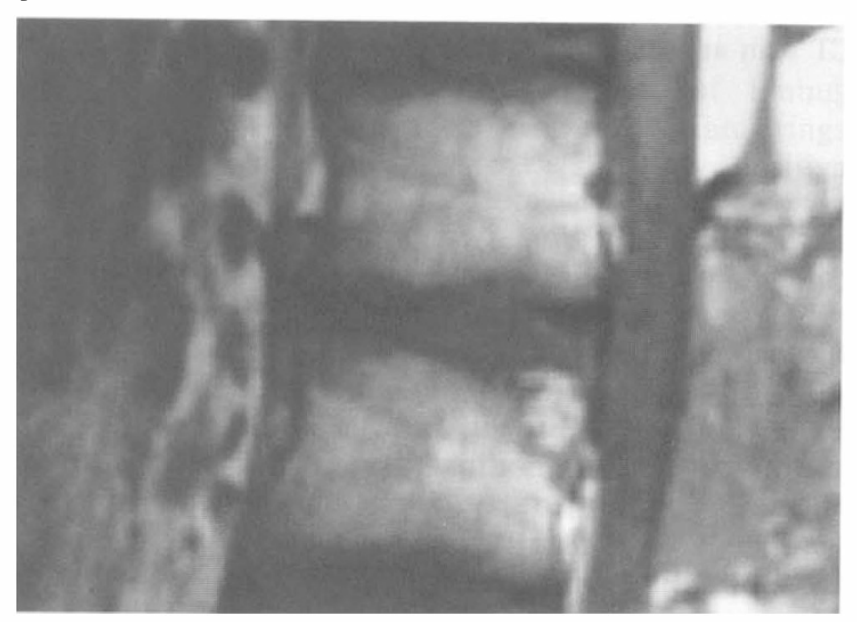

Figure 2 A 15-year-old junior Judo wrestler. (a) preoperative magnetic resonance imaging (MRI) scan showing displaced vertebral corner at proximal part of L2 (type I lesion) (Tl-weighted image, TR 400ms, TE 22ms); (b) preoperative T2-weighted MRI scan (TR, 2000ms, TE, $80 \mathrm{~ms}$ ); and (c) postoperative MRI scan (T1-weighted image, $\mathrm{TR}, 450 \mathrm{~ms}$, TE, $11 \mathrm{~ms}$ ) 
partially explain the exaggerated mechanical signs and hamstrings tightness, but the reason why the latter symptom often lasts for a longer period is entirely unknown.

Preoperative imaging studies should carefully examine for any transaxial and longitudinal extension of the lesion by means of CT and MRI. This is important for the determination of the site of laminotomy, from which the osteocartilaginous mass is safely removed or curetted. Vertebral corner defects are well displayed by conventional radiography and CT as well as MRI. ${ }^{1,3}$ 6,9,10 However, as Swärd et $a l^{11,12}$ and Vincent et $a l^{13}$ indicated, multiple bulges at the posterior intervertebral disc space may be frequent, especially in the elite gymnast. This should be kept in mind when assessing the MRI as well as in selecting the treatment option.

Patients with a small limbus bulge who respond to short-term non-surgical therapy can be treated conservatively. Even if a small osseous fragment is detected, a non-calcified limbus lesion (part of type I, and type NCIII) is not a positive indication for surgery. However, an apparently displaced or avulsed vertebral limbus especially containing a large amount of osseous components, such as types II, CIII, and IV, is not expected to respond well to conservative therapies. If the patient presents with a considerable degree of neurological complications, and the clinical presentation is consistent with the imaging findings, surgical decompression becomes the treatment of choice. Surgical treatment of 29 patients provided us with certain skills particularly with regard to the identification of certain technical requirements to resect the compression lesion safely. The osteocartilaginous lesion may be span longitudinally and transaxially (horizontally), infrequently associated with coexisting disrupted annulus fibrosus posteriorly. A laminotomy opening, without invasion of much of the facet joint medially, should be extended further both in cephalic and caudal directions but, more importantly, the hemilaminectomy approach must not be conducted, from a spinal biomechanical point of view. Lateralised types NCIII and CIII lesions are removed through a unilateral interlaminar window (fenestration). However, for centrally located lesions (types I, II and IV), the optional use of the bilateral interlaminar fenestration approach may be useful and safe. Most patients with limbus vertebral lesions have a tense dural sac and the nerve root at the affected spinal level is considerably compressed from the anterior aspect, together with some adhesions. Following careful dissection between these tissues, the compressive lesion is resected in en bloc and/or in a piecemeal resection fashion. Utmost care must be practised so as not to tug on the lesion posteriorly. Although we did not have such devastating complications, a tense neural tissue is easily damaged causing paralysis. As Epstein et al ${ }^{1,14,15}$ described, the bilateral mallet and downbiting resectioning technique are helpful in the safe removal of the compressive lesion.
For a safer manipulation, we prefer to use a microsurgical technique in order to demarcate the osteocartilaginous lesion using a small diamond burr (outer diameter, 1-2 $\mathrm{mm}$ ) on both sides followed by rather en bloc resection of the mass. Two young adult patients in the present study who required a bilateral hemilaminectomy to eliminate the large anterior mass lesion, subsequently needed a posterolateral fusion. However, because of the young age of the patients, a fusion should be avoided as far as possible, for preservation of the mobile function of the intervertebral disc, especially in subjects with a nearly normal biochemical property of the intervertebral disc at the affected segment as is evident on MRI studies.

In conclusion, the limbus vertebral lesion or avulsed apophyseal ring in young patients produces severe neural entrapment anteriorly with a variable neural compression. An imaging workup should reveal the precise location of the osteocartilaginous compressive lesion prior to surgery. An extended unilateral or bilateral laminotomy approach with the occasional use of a microsurgical technique, not routinely employed for herniated disc excision, is recommended for safe removal of the lesion. A satisfactory surgical outcome in 29 patients was documented in the present study.

\section{References}

1 Epstein NE. Lumbar surgery for 56 limbus fractures emphasizing noncalcified Type III lesions. Spine 1992; 17: 1489 - 1496.

2 White AA, Panjabi MM. The problem of clinical instability in the human spine: the lumbar and lumbosacral spine. In: White AA, Panjabi MM (eds). Clinical Biomechanics of the Spine 2 nd edn. JB Lippincott: Philadelphia 1990, pp. 342-362.

3 Reale RF, Gambacorta D. Calcified lumbar disc protrusion in an adolescent. Surg Neurol 1985; 24: 661-662.

4 Sovio OM, Bell HM, Beauchamp RD, Tredwell SJ. Fracture of the lumbar vertebral apophysis. J Pediatr Orthop 1985; 5: 550. 552.

5 Savini R, Di Silvestre M, Gargiulo G, Picci P. Posterior lumbar apophyseal fractures. Spine 1991; 16: 1118-1123.

6 Goldman AB, Ghelman B, Doherty J. Posterior limbus vertebrae: a cause of radiating back pain in adolescents and young adults. Skeletal Radiol 1990; 19: $501-507$.

7 Ehni G, Schneider SJ. Posterior lumbar vertebral rim fracture and associated disc protrusion in adolescence. J Neurosurg 1988; 68: $912-916$.

8 Takata K. Takahashi K. Hamstrings tightness and sciatica in young patients with disc herniation. J Bone Joint Surg [ Br] 1994; 76: $220-224$.

9 Handel SF, Twiford TW, Reigel DK, Kaufman HH. Posterior lumbar apophyseal fractures. Radiology 1979; 130: 629-633.

10 Dake MD, Jacobs RP, Margolin FR. Computed tomography of posterior lumbar apophyseal ring fractures. J Comput Assist Tomogr 1985; 9: 730732.

11 Swärd L. Hellström M, Jacobson B, Pëterseon L. Back pain and radiologic changes in the thoraco-lumbar spine in athletes. Spine 1990; 15: $124-129$.

12 Swärd L et al. Disc degeneration and associated abnormalities of the spine in elite gymnasts. A magnetic resonance imaging study. Spine 1991: 16: $437-443$.

13 Vincent JM, Baldwin JE, Sims C, Dixon A. Vertebral 'corner' defect associated with lumbar disc herniation shown by magnetic resonance imaging. Spine 1993; 18: 109-113. 
14 Epstein NE, Epstein JA, Mauri T. Treatment of limbus vertebral fractures and spinal stenosis in 4 adolescents and 3 young adults. Neurosurgery 1989; 24: 595-604.
15 Epstein NE, Epstein JA. Limbus lumbar vertebral fractures in 27 adolescents and adults. Spine 1991; 16: 962-966. 